\title{
Passengers' Evacuation in Ships Based on Neighborhood Particle Swarm Optimization
}

\author{
Gan-Nan Yuan, Li-Na Zhang, Li-Qiang Liu, and Kan Wang \\ College of Automation, Harbin Engineering University, Harbin 150001, China \\ Correspondence should be addressed to Li-Na Zhang; ln.zhang201@gmail.com
}

Received 25 November 2013; Accepted 24 January 2014; Published 12 March 2014

Academic Editor: Xiaojie Su

Copyright (C) 2014 Gan-Nan Yuan et al. This is an open access article distributed under the Creative Commons Attribution License, which permits unrestricted use, distribution, and reproduction in any medium, provided the original work is properly cited.

\begin{abstract}
A new intelligent model to simulate evacuation behavior in ships called neighborhood particle swarm optimization is proposed. This model determines the rules of behavior and velocity updating formulas to solve staff conflicts. The individuals in evacuation are taken as particles in PSO and update their behaviors by individual attributes, neighborhood attributes, and social attributes. Putting the degree of freedom movement of ships into environment factor and using the real Ro-Ro ship information and IMO test scenarios to simulate the evacuation process, the model in this paper can truly simulate the behavior of persons in emergency and provide a new idea to design excellent evacuation model.
\end{abstract}

\section{Introduction}

In recent ten years, with the rapid development of shipbuilding industry, the number of high speed and high load of ship has increased sharply. It brings us convenience and wealth and meanwhile increases the frequency of marine perils. Therefore, a security system which can ensure all shipboard personnel evacuation needs to be established urgently. The security system should be matched with set routes, procedures, effective decision support and management, rescue apparatus, and so forth. An optimized design scheme should be considered in ship design, which can enhance passengers' evacuation performance. Even in the situation of safety sailing measure failures, available people assembly and evacuation can also be considered as the final safety shelter to avoid the disaster and the economic loss. Thus, the design of efficient evacuation model has become one of the hottest research areas focusing on ship industry.

In the personnel evacuation progress, dynamic programming for behavior of personnel is needed. With the wide use of evacuation model, more and more research institutions have been dedicated to exploit it. The majority of evacuation models adopt implicit actions, based on function programming, rule-based behavior, or basic behavior of intelligent agent to express evacuation behavior. During the evacuation progress, Helbing's social force model [1] is a typical representation of function programming. Using differential equation to describe the behavior of personnel is too complex to fit large-scale personnel evacuation. Cellular automaton model [2] is a regular basic model, which possesses a good simulation effect but lacks theoretical foundation. Briefly speaking, describing the behavior of personnel in reality by means of several rules is obviously lack of authority. Agent's [3] model is the representation of intelligent agent technology. Izquierdo et al. put forward the idea of applying particle swarm optimization algorithm to simulate human behavior [4]; this algorithm is adopted to large-scale personnel evacuation yet described roughly. Based on above ideas Zheng et al. improve the rules of behavior using the particle swarm optimization algorithm to simulate the process of evacuation [5], while the simulation results lack persuasion because the freedom movement of ship is not taken into consideration. There are many domestic scholars doing research on the passengers' evacuation under the marine environment $[6,7]$. In this paper, on the basis of using the particle swarm optimization algorithm to simulate the process of passengers' evacuation, we improve the simulation system of algorithm and present the simulation method based on the neighborhood particle swarm optimization algorithm. In the meantime we present the update rules of passengers' velocity, the solutions of staff 
conflicts, and take the freedom movement of ship as one of the environment factors. The model in this paper can simulate the process of passengers' evacuation under the condition of ship's freedom movement, and the simulation results show that the algorithm has a high practicability, which provides the ideas for the design of evacuation models with high efficiency and extreme stability.

\section{Particle Swarm Optimization}

The particle in PSO personnel evacuation model can be described as follows: for the ith particle, we consider the candidate solutions of particle swarm optimization algorithm for personnel microscopic evacuation problems as points of space. Even though the solution space can be $N$-dimensional in the application of particle swarm optimization algorithm, while simulating the process of personnel evacuation, we only need two-dimensional space or three-dimensional space. In the whole particle movement process of particle, the attributes of the $i$ th particle can be described by the following three variables:

The current position:

$$
X_{i}=\left(x_{i 1}, x_{i 2}, \ldots, x_{i N}\right) .
$$

The best evacuation individual location reached in the past:

$$
P_{i}=\left(P_{i 1}, P_{i 2}, \ldots, P_{i N}\right) .
$$

The current velocity of the individual:

$$
V_{i}=\left(v_{i 1}, v_{i 2}, \ldots, v_{i N}\right) \text {. }
$$

In the application of standard particle swarm optimization algorithm to simulate the personnel evacuation, the best positions of particles are updated in each iteration. The best position of particles in the whole group is crucial in the searching process; $P_{i}$ represents the best position of each particle. In each iteration, the particles not only learn from the global best position but also learn from the best position of their own. The combination of individual attributes and social attributes for a particle has significant effect on the problems of simulating the personnel evacuation. In the process of simulating the personnel evacuation, the update of particle's velocity and position can be represented by the following formula:

$$
\begin{gathered}
X_{i+1}=X_{i}+V_{i+1}, \\
V_{i+1}=w \cdot V_{i}+c_{1} \cdot \operatorname{rand}()\left(P_{i}-X_{i}\right)+c_{2} \cdot \operatorname{rand}()\left(P_{g}-X_{i}\right) .
\end{gathered}
$$

From the updating formula (5), we can see that the particles obey the inertia of their own firstly to retain part of their own attributes $w \cdot V_{i}$ and then update their behavior according to the best cognitive ability of the environment of their own $P_{i}$; the social cognitive ability of particle movement $P_{g}$ is the global best position $[8,9]$. This mechanism optimization reflects people's actual behavior reasonably.
In the optimization process, particles update their position in each iteration until they reach a special region [10]. In the personnel microscopic evacuation problems described in this paper, the best position of the individual is the safety exit of evacuation. The safety exit can be a single outlet and can also be the selection of multiple exports. When the individual reaches an evacuation exit, we consider that the evacuation individual has found the optimal position, at the same time the current individual withdraws the evacuation sequence, and we record its trajectory and evacuation time. When all the evacuation individuals evacuate safely through the evacuation exit and one process of evacuation has finished, then we record the time of evacuation and the scheme of evacuation.

2.1. Neighborhood Particle Swarm Optimization. Basic particle swarm optimization algorithm has a significant effect on solving the problems about single door evacuation because the targets of all the particles are consistent, moving towards the same evacuation exit [4]. However, when it comes to the complex problems about network, the environment is abstract and there are more than one evacuation exit. In the basic particle swarm optimization algorithm, the particles determine the next position merely according to the individual cognition and social behavior that will cause the problem of all the particles moving toward the best global position. Those problems will lead to large-scale congestion and staff conflicts, which is contrary to the actual evacuation. The behaviors of individuals in actual evacuation mainly depend on the individual cognitive ability for the environment, social behavior, and the behavior of their neighborhood. Therefore, in this paper we will use the improved particle swarm optimization algorithm that contains the neighborhood learning factor to simulate the evacuation, which is out of the limitation of the simple network environment and suitable for the complex evacuation.

In neighborhood particle swarm optimization algorithm, the particles update their velocity and position according to individual behavior, the behavior of the whole group, and the best individual experience in the neighborhood. In the process of evacuation, the neighborhood of a particle is the channel node area which is connected with the position of current particle. Putting the neighborhood learning mechanism into the PSO algorithm not only can simulate the herd behavior of the crowd in the process of evacuation successfully, but also can effectively avoid the problem of all the particles moving toward the local optimal point which could lead to large-scale staff conflicts and congestion. According to the above idea, we modify the velocity updating formula of particle swarm as follows:

$$
\begin{gathered}
V_{i+1}=w V_{i}+c_{1} \text { rand }()\left(P_{\text {best }}-X_{i}\right)+c_{2} \operatorname{rand}()\left(G_{\text {best }}-X_{i}\right) \\
+c_{3} \text { rand }()\left(N_{\text {best }}-X_{i}\right) \\
X_{i+1}=X_{i}+V_{i+1} .
\end{gathered}
$$

$c_{1}, c_{2}$ are learning factors in standard particle swarm optimization algorithm, while $c_{3}$ is neighborhood learning 
factor. $X_{i}$ is the current position of particles. $P_{\text {best }}$ is the best location of particles reached in the past, $G_{\text {best }}$ is the global best position of the particle swarm, and $N_{\text {best }}$ is the best position of the neighborhood of the particle. The application of neighborhood particle swarm optimization algorithm can simulate the behavior of staff conflicts effectively and can solve the problems on the behavior of staff congestion in some extent. In this paper, we consider the circle with the diameter of 0.6 meters as the individual in evacuation, and the neighborhood is the circular area with the evacuation individual as its center and diameter of 2 meters.

2.2. Behavior Process. Firstly, formula (5) presents how the current particle can determine the movement at next moment through the calculation, and then we will judge the feasibility of the movement through the social attributes of populations. If the movement exceeds the environment, it will be restricted and even canceled. The parameter $w$ in formula (5) is

$$
w=0.5+\frac{1}{2 *(\ln (k)+1)} .
$$

This setting for $w$ is according to [11], and $k$ is the iterative number. As the iteration proceeds, $w$ decreases from 1 to 0.5 . This can ensure the global convergence of particles. At the beginning of iteration, the great change of the speed variation can ensure that particles moved to the best position quickly. The change of particles' velocity decreases, with the iteration number increasing, which ensures stronger local convergence when particles arrive at the optimal point. Then $c_{1}=3, c_{2}=2$, and $c_{3}=3$.

Apart from inertia factors, calculation of personnel's velocity is contained by the following factor: environmental layout, individual, and society-related factor. We mainly consider two aspects in evacuation. The first is the choice of exits. In the space of multiple exits, people choose exit generally depending on individuals' ability to perceive information from environment. The second is the queuing phenomenon caused by human-following behavior. In the passenger evacuation progress, congestion occurs frequently.

\section{Velocity Updating Rules}

In the process of actual evacuation, the velocity of people's movement will not increase unlimitedly, whereas the velocity will distribute in a fixed interval. Therefore, the velocity definitely has the upper limit in the PSO model; namely, $V_{i} \leq V_{\max }$. From another perspective, the crowd density of passengers' current space has effect on the moving speed of passengers. According to IMO determination of personnel's speed, this paper takes $1.2 \mathrm{~m} / \mathrm{s}$ as the moving speed of personnel in normal circumstance, and the moving speed will decrease when the crowd density increases. The maximum moving velocity of passengers can be described by the following formula [5]:

$$
V_{\max }= \begin{cases}1.2 \mathrm{~m} / \mathrm{s} & \rho \leq 20 \\ 0.9 \mathrm{~m} / \mathrm{s} & \rho>20 .\end{cases}
$$

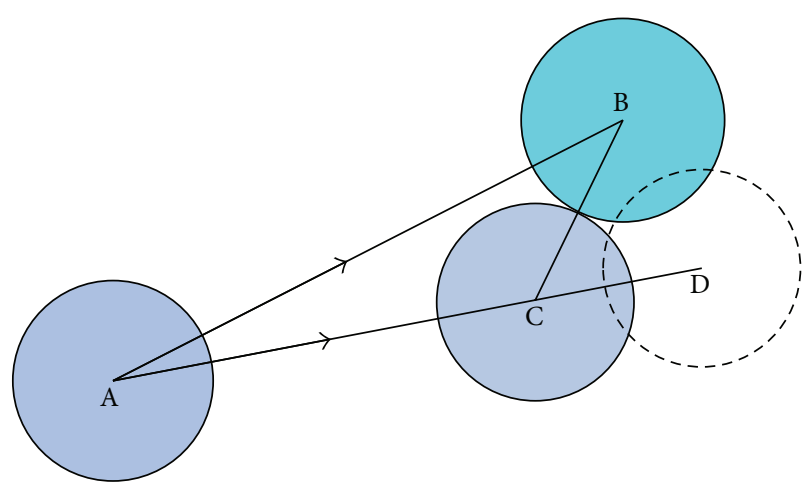

FIGURE 1: Schematic diagram of the staff conflicts.

$\rho$ is defined as the particle's density for the current position; it is the number of people in the circular neighborhood which takes the current particle as center and radius of 2 meters. The individuals in evacuation are taken as circles with the diameter of 0.6 meters. Every particle forms a circular area in which simulates the staff conflicts and updates the velocity. The density of particles changes greatly in different time and different locations. Within the process of evacuation, the positions of passengers and the number of particles around change constantly.

In the evacuation process of particles, each particle has its own unique occupy space that other particles are unable to invade. The occupy space of particles will decrease when the density of particles increases. We use $D$ to represent the diameter of particle's occupy space. The relationship between $D$ and the density of particles can be assumed as follows:

$$
D= \begin{cases}0.6 \mathrm{~m} & \rho<12 \\ 0.4 \mathrm{~m} & 12 \leq \rho \leq 20 \\ 0.3 \mathrm{~m} & \rho>20\end{cases}
$$

Conflicts among the passengers will still exist even though in the application of ideal evacuation process. If a particle's updating position has been occupied by others, the particle will produce a new velocity to avoid staff conflicts (see Figure 1).

Particle A updates its position during the process of evacuation, and its updating position is $\mathrm{D}$, while $\mathrm{D}$ has a confliction of physical location with B. Therefore, the particle A should create a new velocity to make itself move to position $\mathrm{C}$ so as to avoid the conflicts with B.

\section{Passengers' Evacuation Based on PSO Model}

Simulating the process of evacuation with particle swarm optimization algorithm can obtain the behavior characteristics of many individuals successfully. It considers not only the perception ability of individuals on the environment, but also the social attributes of groups in the process of evacuation to make the simulation results more suitable to the real process.

In the process of evacuation, various behaviors of personnel will have an effect on the evacuation velocity of 
individuals, for instance, aggregation behavior, individuals in evacuation move towards to the center of population or move towards to the position with fewer people; people will maintain a certain distance between each other, which means a person will have the same velocity with other persons who are nearby, people will obey the evacuation signal, and so forth. A person's individual property such as age, gender, and health status will have effects on their walking speed. An excellent simulation model can reflect the influence of personnel's psychological factors such as pain, fear, and negative emotions. People's cognition degree of sound, environment, and facilities will have influences on the process of evacuation. We can establish the comprehensive consideration of the above situation with the application of the fuzzy comprehensive evaluation. Simultaneously, it will have great influence on the evacuation behavior of population if passengers' psychological effects are considered too much. We suppose that the passengers have slight psychological effects in the research process so that they will not lose the behavior of evacuation because of panic caused by emergencies.

For the selection of evaluation function, it is expressed as the distance between the person and the nearest evacuation exit to him or her. $X$ is the position of current particle and $E$ is the collection of evacuation exit:

$$
F(X)=d(X, E)=\min \{d(X, e), e \in E\} .
$$

Function $F$ is obviously a nonlinear function in most conditions. The procedures of applying the PSO model to solve the problems of passengers' evacuation can be described as follows.

Step 1. Set the number of particles (the summation of individuals in evacuation). Distribute the initial position randomly. Set the number and the positions of evacuation exits.

Step 2. Set the value of inertia factor $w$, learning factors $c_{1}$ and $c_{2}$, and neighborhood learning factor $c_{3}$. Record all the particles in current position and velocity.

Step 3. Calculate the value of fitness function for all the particles. If the distance between a particle and a safety exit is 0 , then the current particle evacuates successfully and this particle is no longer retained in the following process of evacuation. Record the particles whose distance to the safety exits is more than 0 .

Step 4. Update particles' velocity according to formula (6); judge whether the condition of the velocity's upper limit is satisfied. If it is satisfied, update the velocity as usual; if it is not satisfied, the velocity's upper limit is assigned to the current particle.

Step 5. Judge whether the particles' conflicts exist; namely, whether the movement direction of current particle has been occupied. If it is not occupied, update the position as usual; if it is occupied, generate the velocity increment randomly and update the position.
Step 6. Judge if all the particles have been reached to the safety exits: if it is satisfied, go to Step 7; else go to Step 3 .

Step 7. When the process of evacuation is finished, output the evacuation time and the dynamic graph of evacuation.

The algorithm will finish if the individuals in evacuation have evacuated successfully, and then output the evacuation time.

\section{Simulations}

In the following context, we will discuss the effectiveness of the PSO evacuation model proposed in this paper through the numerical experiment. Firstly, we set the parameters of neighborhood particle swarm optimization algorithm to simulate the problem of evacuation, $w=0.5+0.5 /[\ln (k)+1]$, $w$ varies from 1.0 to 0.5 , and $c_{1}=3, c_{2}=2$, and $c_{3}=3$. The iteration step $T=0.5$ [12].

This research selects a cabin with the 2 meters wide door as the research object to simulate the process of microscopic passengers' evacuation by the software of Visual Studio 2010.

Figure 2 shows that individuals in evacuation distribute randomly in the evacuation space at the beginning of evacuation. The individuals that are close to the safety exits of evacuation can find the evacuation plan quickly, and the individuals that are far from the safety exits of evacuation can also find the direction of evacuation fleetly. With the learning strategy of particle swarm, the individuals in evacuation can make quick and effective evacuation judgments in order to find the best evacuation routes which are suitable to their current locations.

5.1. The Influence of Doors' Dimension for the Process of Evacuation. Different sizes of the door have quite different evacuation capacities. Obviously, the larger size can get the well effect of evacuation. But in the period of ship design, the size of doors cannot be randomly designed due to the limitation of space and size of ship. Hence, the most appropriate size for doors needs to be designed under limitation of surroundings and conditions to improve the evacuation capacity to the ultimate extreme. The influence of doors' dimension for the process of evacuation is shown in Figures 3 and 4.

By fixing the number of escaping people at 200 as initial value, Figure 3 shows the changing curve of egress time of 200 people with the size of the door increasing. From the figure we can see obviously that, with the size of the door increasing, the evacuation time is effectively reduced. When the size is larger than 4 meters, it has little effect on the evacuation time. It shows that, in this condition, the size of the door has lower influence on the evacuation time, and there exists the best threshold for the size.

For different sizes of the door, with the number of people increasing, the changing curve of the evacuation time is shown in Figure 4 . When the door width is set to 2, 3, 4 , and 6 meters, respectively, the evacuation time significantly increases with the number of peopleincreasing. When 


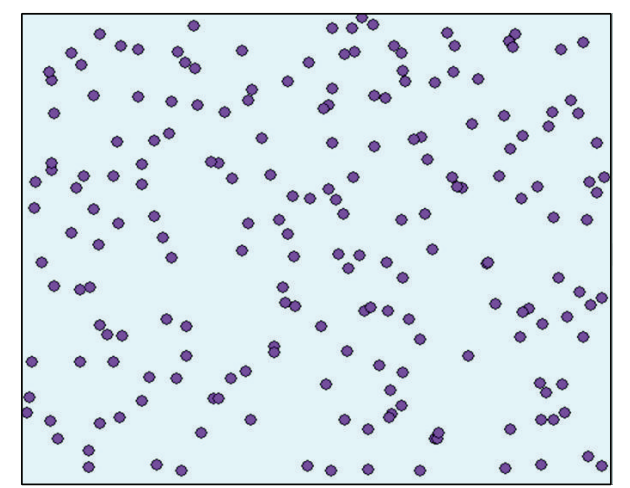

(a)

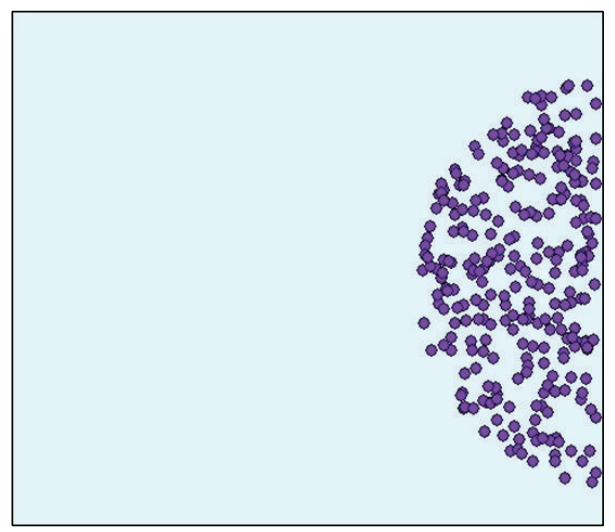

(c)

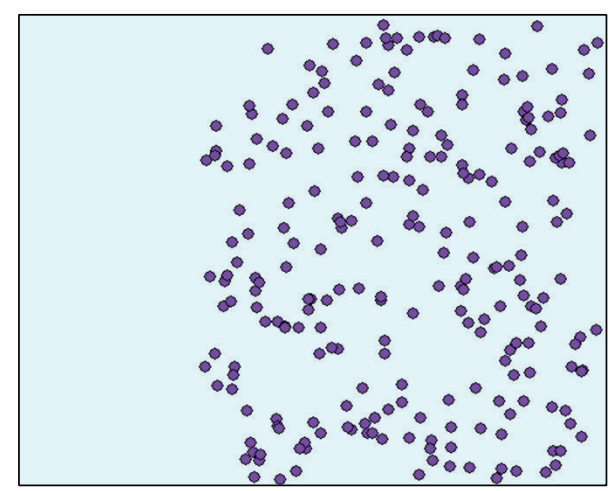

(b)

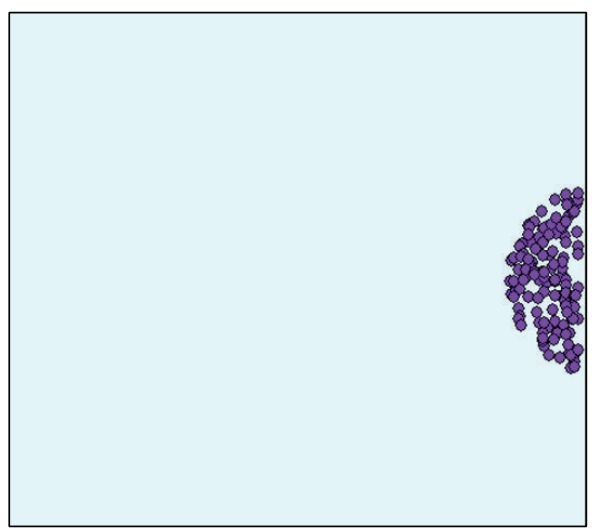

(d)

FIGURE 2: The distribution of the evacuation of people in different time.

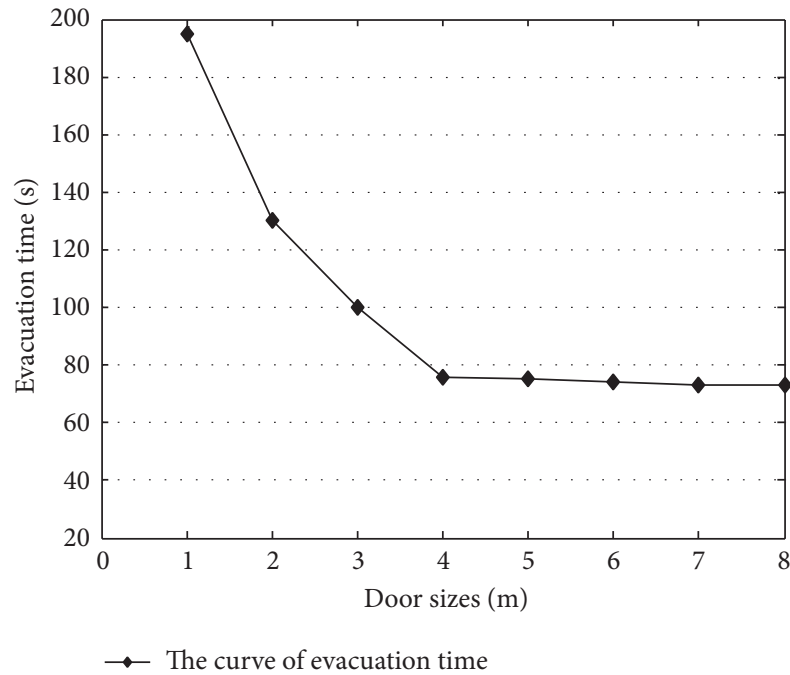

FIGURE 3: Effect of the door size on evacuation time.

the door width is set to 4 or 6 meters, the difference of the evacuation time is small. But when setting the dimension for 2 or 3 meters, the difference is huge. The evacuation time for 2 meters is nearly 1.5 times longer than 6 meters of the doors' dimension. However when the doors' dimension is over

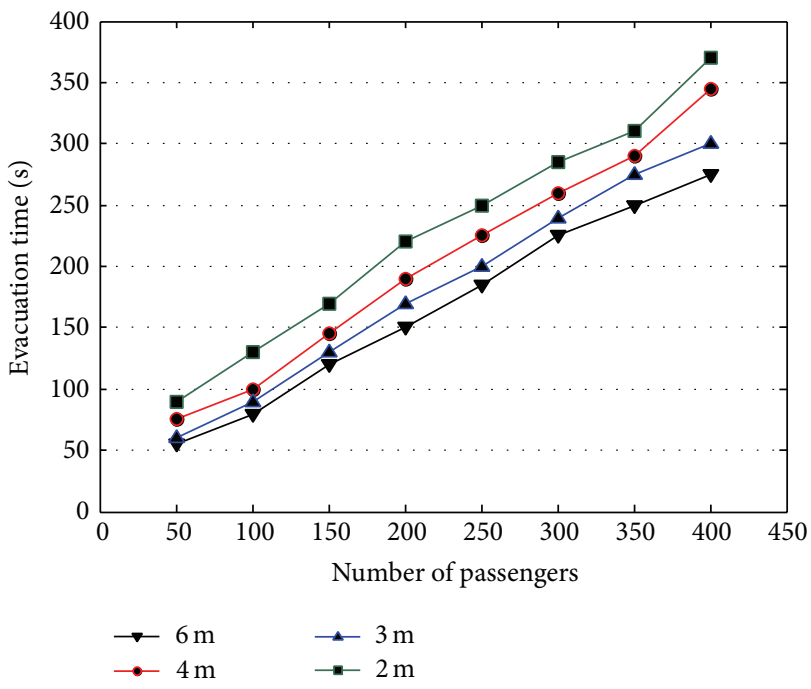

FIGURE 4: Effect of door size and number of people on evacuation time.

4 meters, the difference was not significant. This is verified by Figure 3. In the premise of the suitable designing dimensions of the ship, there exists the best dimension for evacuation. 


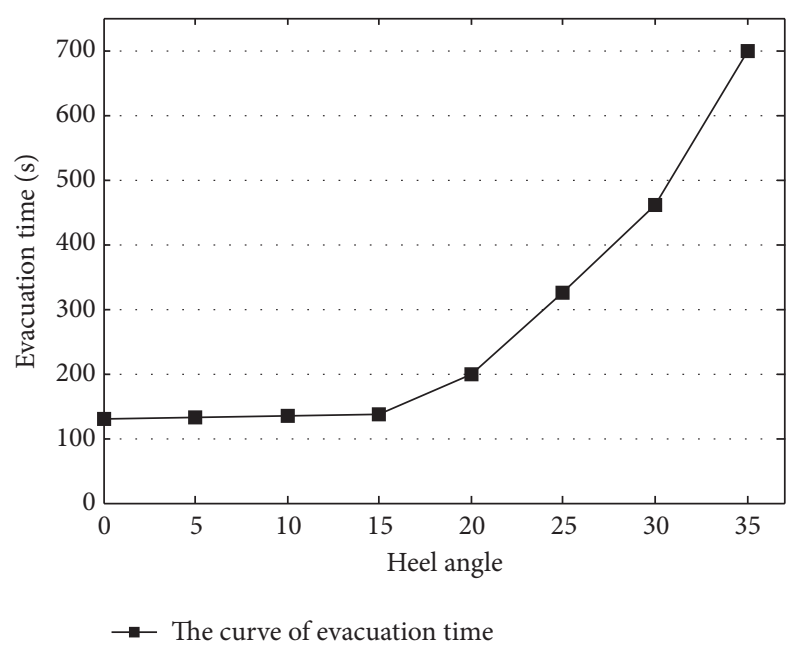

(a) Heel conditions

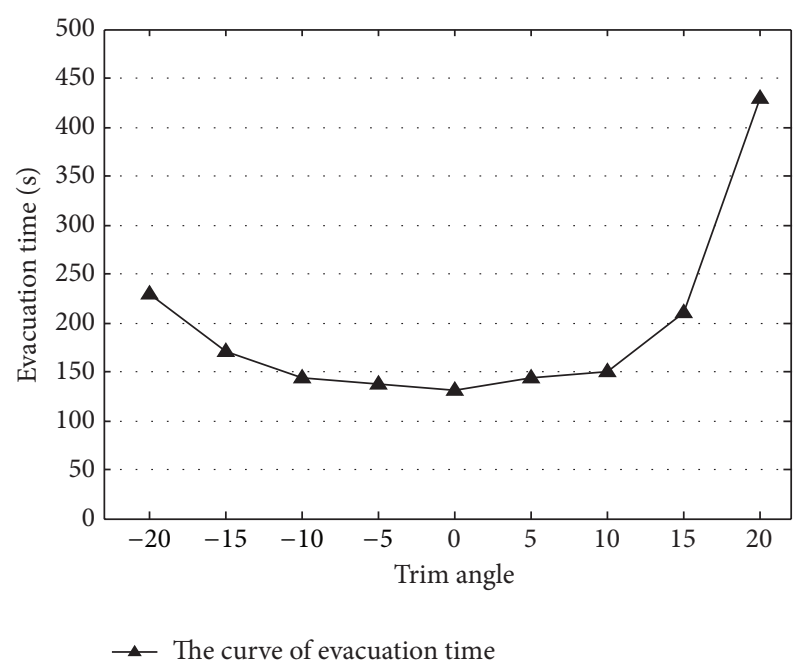

(b) Trim conditions

FIgURE 5: The time influences of the ship's heel and trim.

If the doors' dimension is fixed, the evacuation time obviously increases with the increasing number of people.

5.2. The Influences of the Ship's Heel and Trim. An experiment is conducted to test the time influences of the ship's degree of freedom. It is conducted under the condition that the angle of ship's heel changes from 0 to 35 degrees and the angle of ship's trim changes from -20 to 20 degrees. The number of passengers is fixed as 200 as well. Figure 5 reflects the influences of the ship's DOF (degree of freedom) movement on passengers' evacuation time. Figure 5(a) is in the condition of ship's heel. When the angle of ship's heel changes from 0 to 15 degrees, the effect on passengers' behavior of evacuation is very little. However, the influence is enhanced gradually when the angle of ship's heel is more than 20 degrees, and when it turns to more than 30 degrees, the evacuation time of the whole 200 persons is 3-6 times of that in steady state. The above fact proves that it is not of any significance without the consideration of ship's movement in the simulation of passengers' evacuation process.

Figure 5(b) exhibits the influences of the ship's trim on passengers' evacuation time. When the angle of ship's trim changes from -15 to 15 degrees, it has fewer effect on passengers' evacuation time, while when the angle of ship's trim increases to 20 degrees, it has a tremendous impact on individuals in evacuation. The angle of ship's trim can also be interpreted as the angle of lateral inclination. With the increasing of trim's angle, the left-right force among the persons increases, which will greatly hinder the movement of passengers; the conflicts among the individuals and the congestion caused from the conflicts are increased obviously. However, in the condition of ship's heel, it is the pre-post force that increases, the influence of pre-post force caused by ship's heel will be significant when the crowd density is high in evacuation. If the distribution of individuals is not intensive, the force caused by ship's heel will have a slight effect on the population movement, but it will seriously affect the behavior of individuals in evacuation. The increasing of the heel's angle and the inclination of walking plane will increase the difficulty for the individuals in evacuation greatly when they are walking and the same time it depends on the individual differences in ability. It will make the whole process of evacuation more difficult when the angle of ship's heel and trim increases. Thus, ship's heel and trim are the main factors that influence people's behavior in evacuation.

5.3. The Process of Simulating the Evacuation System with Multiple Doors. Also in the evacuation space of $25 \times 25 \mathrm{~m}$, four evacuation exits are settled in the evacuation space. In the designation of ships, the size of the door is restricted, so in order to match the actual situation, the width of the door is set to 2 meters. The initial distribution of persons is random and the crowd is divided into three parts, the youth, the elderly, and the children. Meanwhile, all of them are given different walking speed and initial speed. The red circles represent the elderly, purple circles represent the children, and yellow circles represent the youth. The distributions of the crowd at $0 \mathrm{~s}, 40 \mathrm{~s}, 80 \mathrm{~s}$, and $120 \mathrm{~s}$ can be seen from Figure 6; the results of simulation clearly show the aggregation behavior of adults and children, as well as the walking condition of elderly people.

The results can be seen from Figure 6 that the algorithm of PSO model is still remarkable on the evacuation system with multiples doors. The initial distribution of persons in the space is random. But with the evacuation process continuing, the passengers find their own evacuation directions immediately. The behavior of group is significant and the staff conflicts are lower in the process of evacuation. Figure 7 shows the curve of passengers' evacuation time under the condition of four doors with the increasing number 


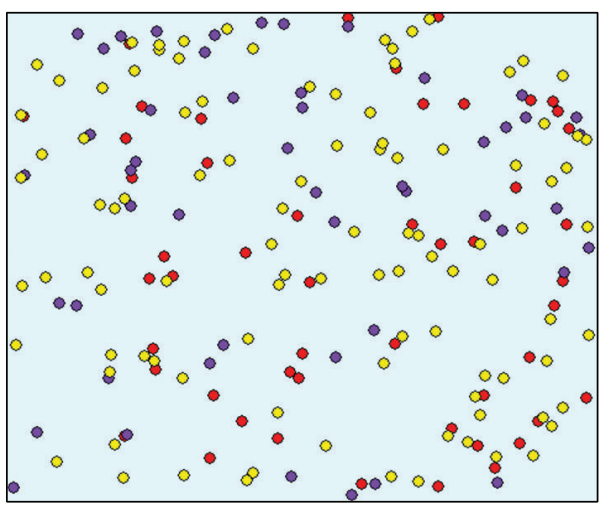

(a)

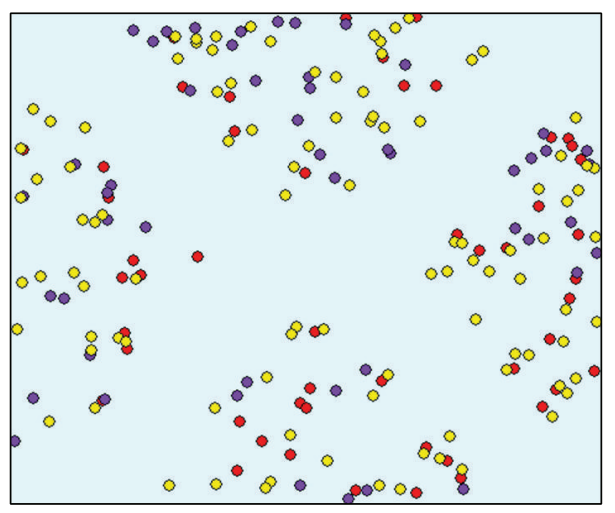

(c)

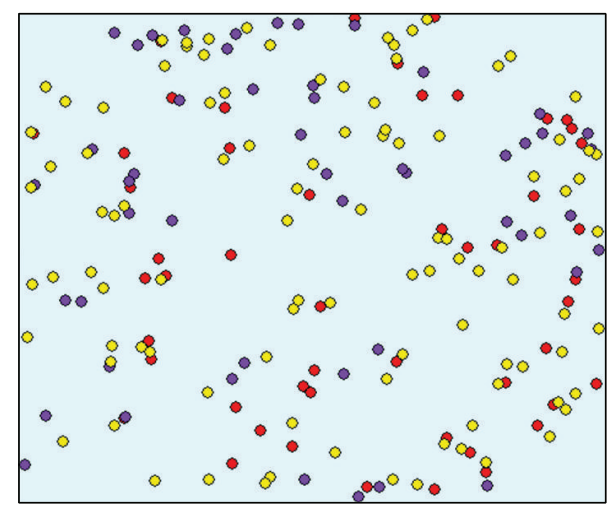

(b)

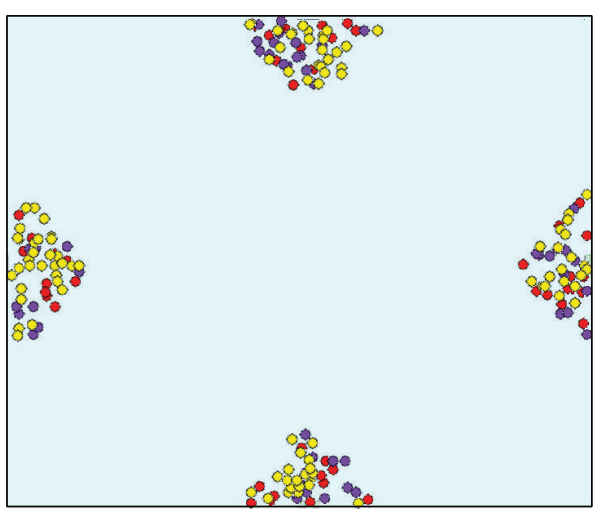

(d)

Figure 6: The distributions of the persons in the case of four doors

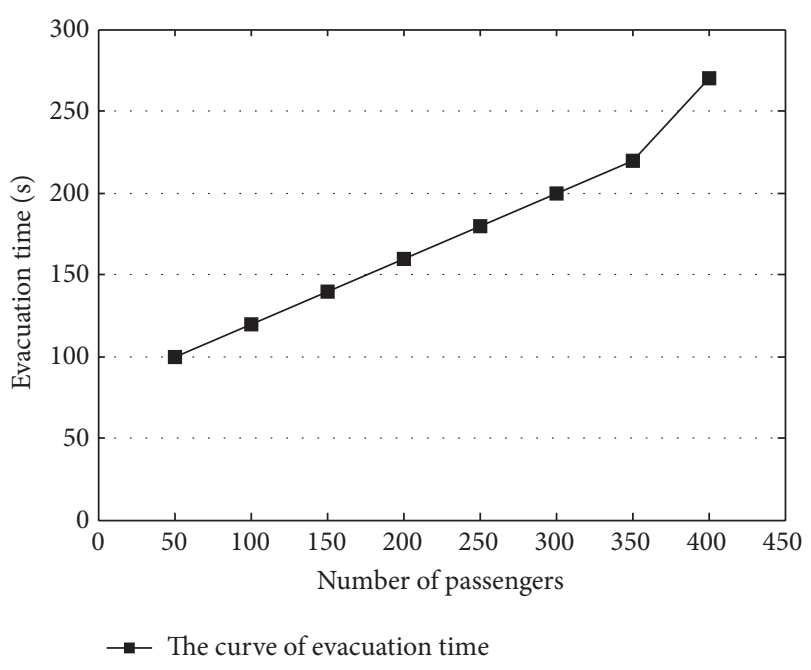

FIGURE 7: The relationship between evacuation time and the number of passengers.

of passengers, and the results reflect that the evacuation time increases significantly when the number of passengers increases.
We explore the influence of the ship's DOF movement for the conditions of four doors with the fixed 200 passengers. The angle of ship's heel is set to change from 0 degrees to 35 degrees and the angle of ship's trim changes from -20 degrees to 20 degrees. Figure 8 shows the effects of ship's DOF movement on the evacuation time. The curve shape of evacuation time in the condition of four doors is roughly equal to that of single door. But the whole evacuation time decreases because the evacuation space is larger, and the staff conflicts are lower in the condition of four doors. Meanwhile, the more the evacuation exits are, the stronger the evacuation capacity of the cabin is. If we only take the evacuation ability of a cabin into consideration, the evacuation time should be a quarter of that in the condition of single door. However, the actual situation is not the same as what we have mentioned above. It is because the personnel microscopic behavior simulation algorithm based on the PSO model takes the individuals' movement into consideration. The congestion, conflicts, and queuing phenomenon of persons will increase the whole evacuation time, so that it will make the simulation results more realistic.

5.4. IMO Test 8: The Simulation Test of Personnel Convection. In the process of passengers' evacuation in marine environment, the phenomenon of personnel convection is always 


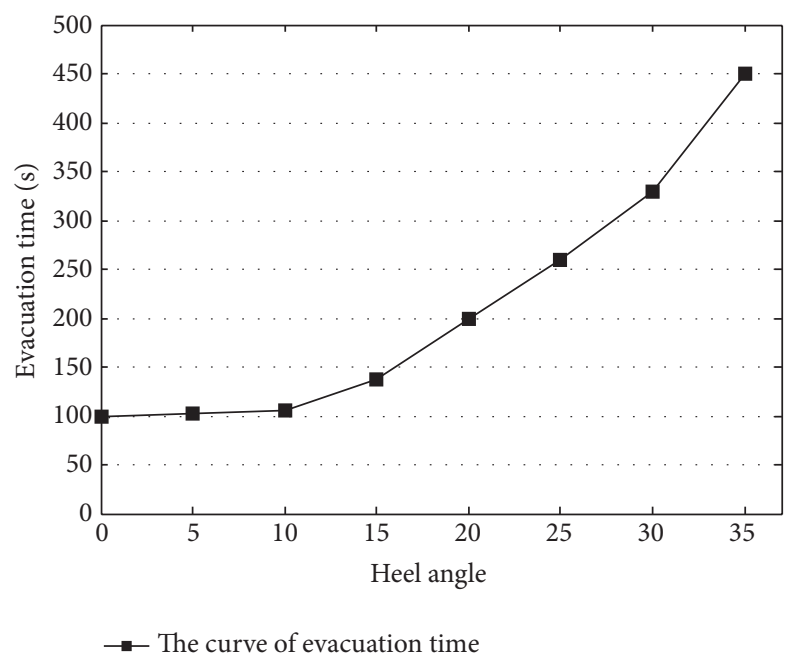

(a) Heel

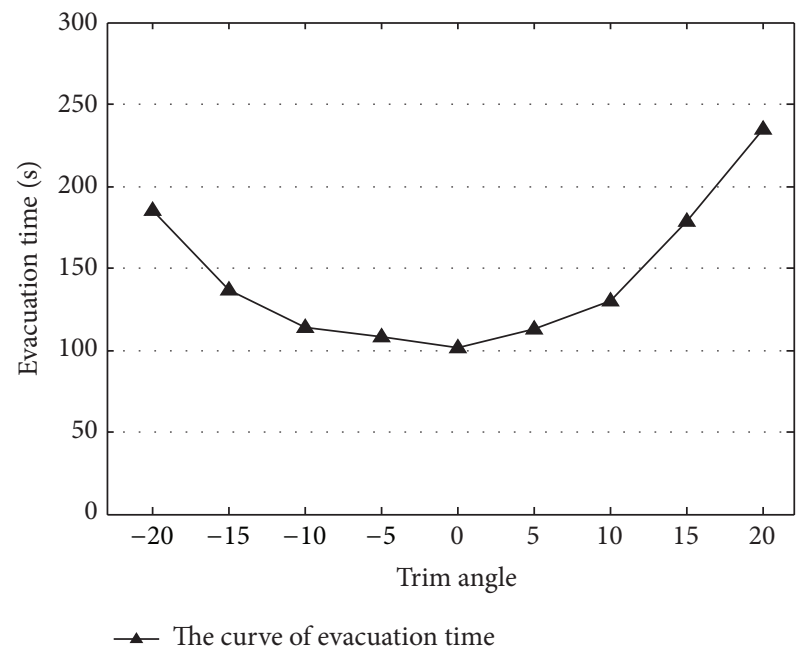

(b) Trim

FIGURE 8: The time influences of the ship's heel and trim in the case of four doors.

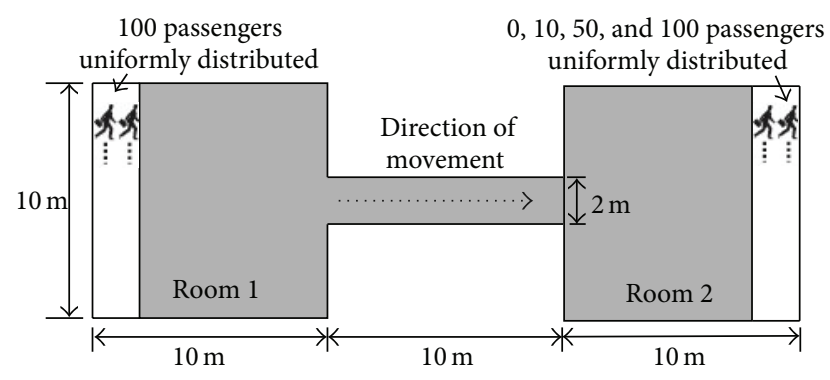

FIGURE 9: Simulation environment of test 8.

accompanied by the evacuation process, and it is one of the main factors affecting personnel behavior. Then we will simulate the scene of IMO convection test with the PSO evacuation model. The scenes of convection test regulated by IMO are as follows: two evacuation groups are arranged in the adjacent compartments, respectively, and there is a passage which connects the two compartments. People in room 1 need to move to room 2 , and people in room 2 need to move to room 1 . The initial number of individuals in room 1 is 100 [13], and the characteristics of the individuals are 30-50-year-old evacuation persons. The passage is 10 meters long and 2 meters wide. The phenomenon of convection occurs in a large range when passengers in room 2 are trying to move to room 1 , and the initial number of individuals in room 2 is set, respectively, as $0,10,50$, and 100 (see Figure 9). The results of simulation are shown in Table 1; the evacuation time significantly increases when the number of the convective population increases. Figure 10 shows the process of evacuation as the time goes by under the condition when the number of convection population is 100 .

The experiment results reflect the fact that the evacuation time will increase greatly when the number of individuals
TABLE 1: The contrast of PSO and EVI.

\begin{tabular}{lcc}
\hline Parameters & \multicolumn{2}{c}{ Evacuation time (s) } \\
Convection passengers & PSO & EVI \\
\hline 0 & 83.7 & 88.9 \\
10 & 92.6 & 125.6 \\
50 & 136.9 & 229.1 \\
100 & 215.1 & 327.9 \\
\hline
\end{tabular}

in evacuation in room 2 increases. In the meanwhile, in the process of evacuation, the personnel convection is largely reduced because of the attributes of social learning of PSO model. We can see from Table 1 that the research results in this paper are significantly enhanced [14] compared with other similar researches, and the whole evacuation time has greatly improved.

\section{Conclusions}

This paper mainly studies how to simulate the process of passengers' evacuation under the circumstance of emergency evacuation and establishes the simulation model of the passengers based on the neighborhood particle swarm optimization algorithm. In this paper, we consider the particles in the swarm as the individuals in evacuation, and the behaviors of the particles are directed by their own learning abilities, the perception abilities of environment, and the social attributes, which can simulate the real process of evacuation. We use the scene of IMO convection test as well as the real data of a ship to test the properties of the model presented in this paper. The simulation results show that the model of passengers' behavior based on PSO algorithm is very effective and has a strong promotional value, and it provides ideas for designing a stable and efficient evacuation behavior model. 

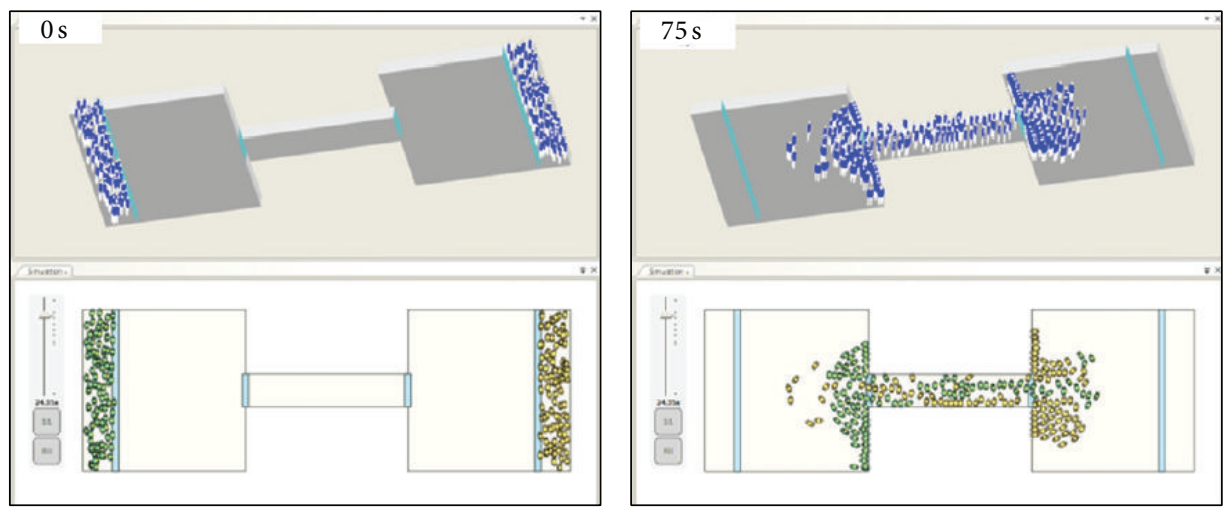

(a)


(b)

FIGURE 10: Simulation effects of test 8 with all 100 persons in room 1 and room 2 .

\section{Conflict of Interests}

The authors declare that there is no conflict of interests regarding the publication of this paper.

\section{Acknowledgment}

This work was supported by the National Natural Science Foundation of China (nos. 51009036 and 51109045).

\section{References}

[1] D. Helbing, I. Farkas, and T. Vicsek, "Simulating dynamical features of escape panic," Nature, vol. 407, no. 6803, pp. 487490, 2000.

[2] C. Burstedde, K. Klauck, A. Schadschneider, and J. Zittartz, "Simulation of pedestrian dynamics using a two-dimensional cellular automaton," Physica A, vol. 295, no. 3-4, pp. 507-525, 2001.

[3] S.-Y. Liao and J.-H. Dai, "Design and implementation of communication system in agent-based distributed simulation," Journal of System Simulation, vol. 18, no. 4, pp. 1006-1014, 2006.

[4] J. Izquierdo, I. Montalvo, R. Pérez, and V. S. Fuertes, "Forecasting pedestrian evacuation times by using swarm intelligence," Physica A, vol. 388, no. 7, pp. 1213-1220, 2009.
[5] Y. Zheng, J. Chen, J. Wei, and X. Guo, "Modeling of pedestrian evacuation based on the particle swarm optimization algorithm," Physica A, vol. 391, no. 17, pp. 4225-4233, 2012.

[6] W. Yu, "Research on the evacuation route of passage in ship based on shortest path algorithm," Chinese Journal of Ship Research, vol. 3, no. 2, pp. 16-20, 2008.

[7] G. Xu, Study of the simulation of evacuation dynamics [Master Degree Thesis], Southwest Jiaotong University, 2003.

[8] C. P. Salomon, G. Lambert-Torres, L. E. B. da Silva, M. P. Coutinho, and C. H. V. de Moraes, "A hybrid particle swarm optimization approach for load-flow computation," International Journal of Innovative Computing, Information and Control, vol. 9, no. 11, pp. 4359-4372, 2013.

[9] H. Jabeen and A. R. Baig, "GPSO: a framework for optimization of genetic programming classifier expressions for binary classification using particle swarm optimization," International Journal of Innovative Computing, Information and Control, vol. 8, no. 1, pp. 233-242, 2012.

[10] B. S. Ahmed, K. Z. Zamli, and C. P. Lim, "Constructing a tway interaction test suite using the particle swarm optimization approach," International Journal of Innovative Computing, Information and Control, vol. 8, no. 1, pp. 431-451, 2012.

[11] Y.-X. Jin, H.-Z. Cheng, J.-Y. Yan, and L. Zhang, "New discrete method for particle swarm optimization and its application in transmission network expansion planning," Electric Power Systems Research, vol. 77, no. 3-4, pp. 227-233, 2007. 
[12] S. Ha, N.-K. Ku, M.-I. Roh, and K.-Y. Lee, "Cell-based evacuation simulation considering human behavior in a passenger ship," Ocean Engineering, vol. 53, pp. 138-152, 2012.

[13] E. Vanem and R. Skjong, "Designing for safety in passenger ships utilizing advanced evacuation analyses-a risk based approach," Safety Science, vol. 44, no. 2, pp. 111-135, 2006.

[14] D. Harwood and A. Farrow, "Validation of reported events from a vertical chute marine evacuation study," Applied Ergonomics, vol. 43, no. 6, pp. 1105-1109, 2012. 


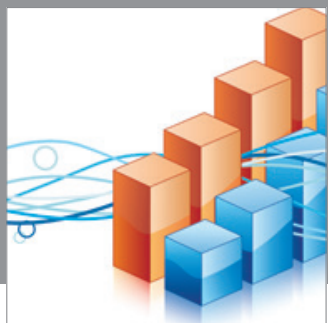

Advances in

Operations Research

mansans

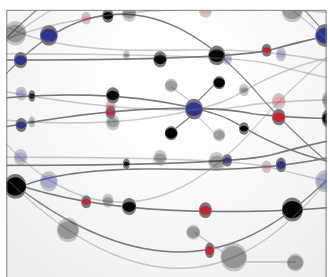

The Scientific World Journal
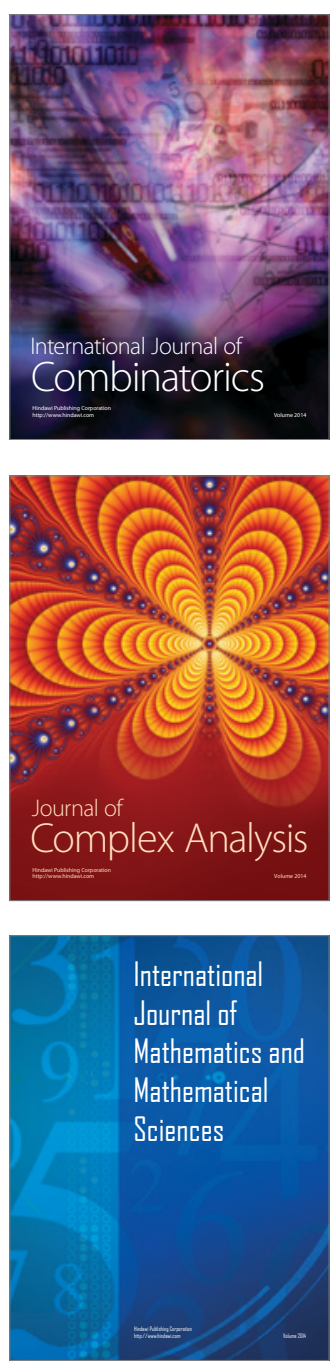
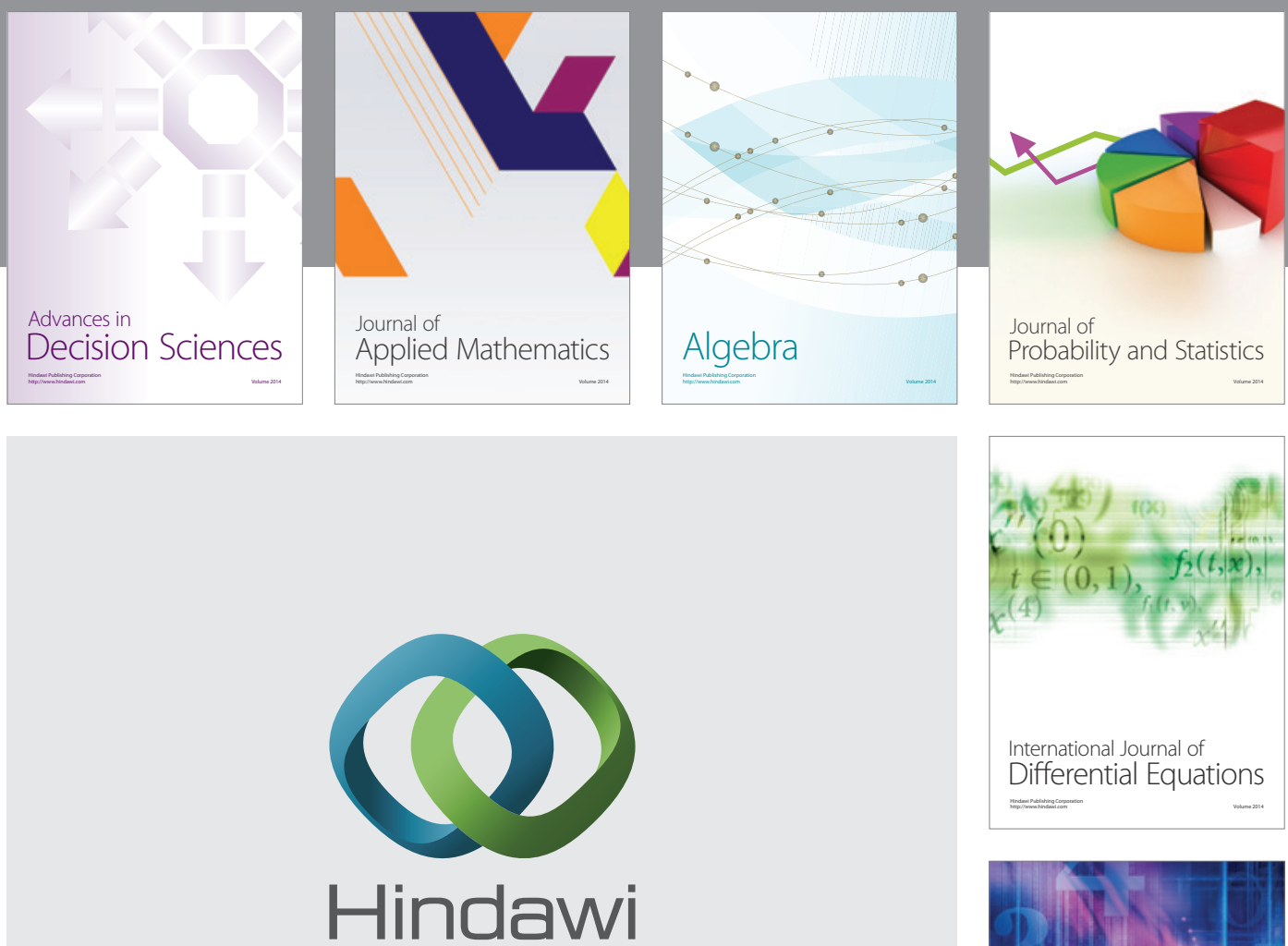

Submit your manuscripts at http://www.hindawi.com
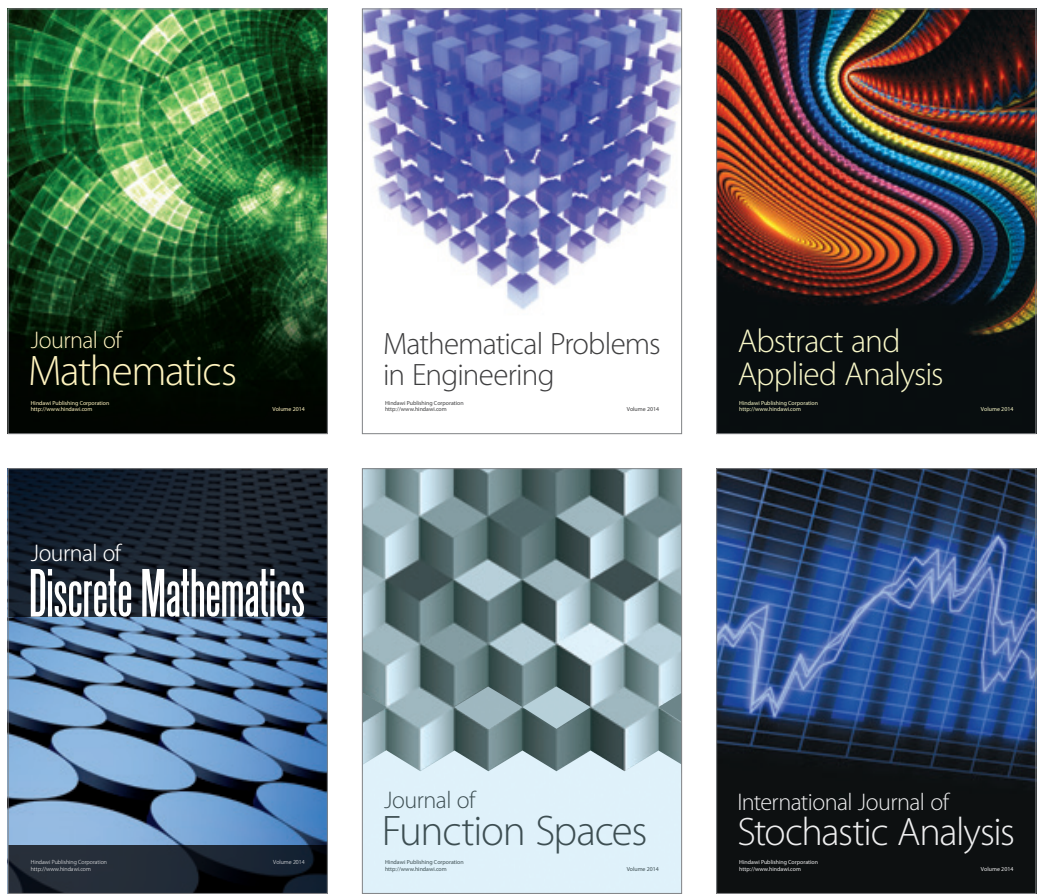

Journal of

Function Spaces

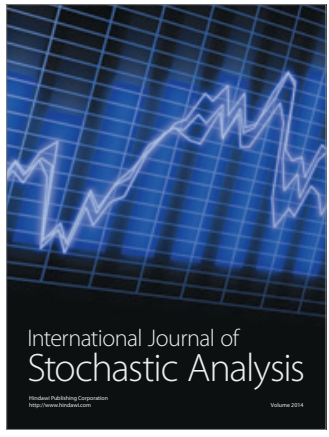

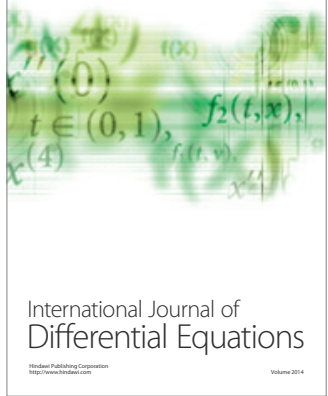
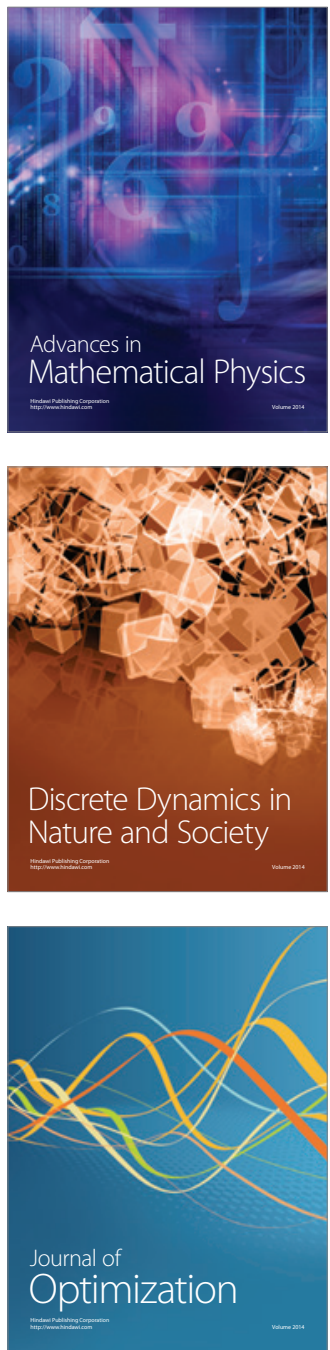